\title{
BMJ Open Effect of peer support on patient anxiety during the coronary angiography or percutaneous coronary intervention perioperative period: a protocol for a systematic review and meta-analysis of randomised controlled trials
}

\author{
Shuo Qin (D) , ${ }^{1}$ Yanmei Gu, ${ }^{1}$ Tianyu Song ${ }^{2}$
}

To cite: Qin S, Gu Y, Song T. Effect of peer support on patient anxiety during the coronary angiography or percutaneous coronary intervention perioperative period: a protocol for a systematic review and meta-analysis of randomised controlled trials. BMJ Open 2020;10:e031952. doi:10.1136/ bmjopen-2019-031952

- Prepublication history for this paper is available online To view these files, please visit the journal online (http://dx.doi. org/10.1136/bmjopen-2019031952).

Received 30 May 2019 Revised 24 February 2020 Accepted 10 March 2020

Check for updates

(c) Author(s) (or their employer(s)) 2020. Re-use permitted under CC BY-NC. No commercial re-use. See rights and permissions. Published by BMJ.

${ }^{1}$ College of Nursing, Hebei University of Chinese Medicine, Shijiazhuang, Hebei, China

${ }^{2}$ Faculty of Health and Medical Sciences, The University of Adelaide, Adelaide, South Australia, Australia

Correspondence to Professor Yanmei Gu; yanmei.gu@mail.utoronto.ca

\section{ABSTRACT}

Introduction The purpose of this study is to investigate the effect of peer support on patient anxiety during the perioperative period of coronary angiography or percutaneous coronary intervention (PCl).

Methods and analysis We will search the following databases (PubMed, Web of Science, EMBASE, Cochrane Library, CINAHL, Clinicaltrials.gov, WHO International Clinical Trials Registry Platform, Google Scholar, Chinese National Knowledge Infrastructure, Chinese Science and Technology Periodicals Database, Chinese BioMedical Database and Wanfang Data) from the date of database inception to January 2019. Only randomised controlled trials will be included. For the data analysis, we will use RevMan V.5.3.5 software to evaluate the risk of bias, and the heterogeneity will be investigated using the $Q$ statistic and $\mathrm{P}$ index. Additionally, the Grading of Recommendations Assessment, Development and Evaluation system will be used to assess the quality of evidence.

Ethics and dissemination № ethics approval will be required since this is a systematic review of published studies. We aim to report information regarding the effects of peer support on patient anxiety during the perioperative period of coronary angiography or PCl. This systematic review and meta-analysis will be submitted to a peerreviewed journal for publication.

PROSPERO registration number CRD42019123290.

\section{INTRODUCTION}

Cardiovascular disease (CVD) is one of the most common non-communicable diseases worldwide. $^{1}$ It has high morbidity and mortality. ${ }^{2}$ It not only seriously threatens human health but also results in heavy social and economic burdens. ${ }^{3}$ CVD-related deaths in Europe and the USA account for $10 \%$ of all deaths. ${ }^{4}$ The WHO predicts that the mortality rate of coronary heart disease will reach $13 \%$ by $2030 .^{2}$ At present, there are approximately 11 million patients with coronary heart disease in China. ${ }^{5}$ CVD ranks first among all

\section{Strengths and limitations of this study}

- This will be the first systematic review and metaanalysis to update estimates of the ability of peer support to reduce perioperative anxiety in patients with coronary angiography or percutaneous coronary intervention.

- Two reviewers (SQ and TS) will independently review the full articles, data extraction and risk of bias assessment.

- Only randomised controlled trials will be included in this study.

- Because only English and Chinese studies will be included, language bias exists.

causes of mortality, accounting for more than $40 \%$ of residents' disease-related deaths, and the morbidity and mortality rates are continuously rising. ${ }^{6}$ From 2004 to 2015, hospitalisation expenses related to acute myocardial infarction increased by $30 \%$. The growth rate of total hospitalisation expenses was higher than that of gross domestic product. ${ }^{7}$

Percutaneous coronary intervention (PCI) is the main treatment method for coronary heart disease; an average of 800000 patients with CVD in Europe and 1 million patients with CVD in the USA per year undergo PCI. In China, $560000^{8}$ cases of coronary heart disease have been treated by PCI, with a success rate of $91 \%-97 \% .^{9}$ Among medical services, PCI has the advantages of being minimally invasive, having a short recovery cycle and causing less pain. ${ }^{10}$ Coronary angiography is the gold standard for the diagnosis of coronary heart disease and is a necessary examination before PCI. ${ }^{5}$ However, for patients, heart-related surgery, regardless of its minimal invasiveness, is a stressor that causes severe physical and 
mental reactions. Many factors affect the emotional state of patients during the perioperative period (both before and after the procedure), ${ }^{56}$ such as a lack of knowledge about the interventional therapy and uncertainty about safety; these factors can easily cause perioperative anxiety in patients with PCI. ${ }^{11}$ Approximately $20 \%$ of patients in China have anxiety after PCI, ${ }^{12}$ and anxiety is an important factor in cardiovascular events. Studies have shown that severe anxiety is associated with decreased immunity and changes in cardiovascular function, ${ }^{13}$ which can cause symptoms, such as arrhythmia and vascular inflammation, and may lead to deterioration of the coronary artery condition. ${ }^{14-16}$ Anxiety leads to a rapid heartbeat, high levels of hypertension, an increased possibility of arrhythmia, ${ }^{17}$ delayed wound healing, an increased risk of infection, electrolyte and fluid imbalance, changes in sleep patterns, increased pain after surgery, and delayed discharge, ultimately reducing patient satisfaction with medical and nursing care.

In the theory of social learning theory, peer support refers to a type of education that can offer greater empathy through the sharing of knowledge and experience within specific groups by using the influence of peers and conveying positive attitudes and ideas with one another (thereby reducing heart attacks and anxiety and diminishing the possibility of readmission).${ }^{18}$ Patients learn how to cope with disease through their peers' experiences. Peer support was first used in the fields of AIDS and reproductive health. ${ }^{20}{ }^{21}$ Recent studies have confirmed that in terms of emotions and information, peer support plays a very important role in life behaviour changes and the health of patients undergoing coronary artery bypass grafting. ${ }^{19}$

Important aspects of nursing are comprehensively evaluating patients' physical and mental statuses during the perioperative period, effectively reducing patients' stress responses and alleviating patients' anxiety. Current studies suggest that the main measures for alleviating perioperative anxiety associated with PCI are psychological nursing, evidence-based nursing and music therapy. ${ }^{22-26}$ The main source of these interventions is medical staff, which presents a serious challenge given the current situation of medical resources in China. Bandura's self-efficacy theory suggests that peer support can enhance individual selfefficacy; patients with higher levels of self-efficacy are relatively more confident in participating in decision-making and regulating their emotional state.

In the current study, we will retrieve previously published studies on peer support interventions designed to cope with perioperative anxiety in patients with heart procedures and analyse them with RevMan V.5.3.5 to draw a relatively reliable conclusion.

\section{METHODS AND ANALYSIS}

This article will be based on the Preferred Reporting Items for Systematic Review and Meta-Analysis (PRISMA) protocols 2015 statement.

\section{Eligibility criteria}

Study design

Randomised controlled trials (RCTs) will be enrolled regardless of the length of the peer support intervention.

\section{Participants}

Participants in the enrolled articles should be in hospital with the following characteristics: (1) older than 18 years of age; (2) a clear description of the diagnosis; (3) a description of having provided informed consent; and (4) a clear description of the procedures involved. Those (1) undergoing emergency coronary angiography or PCI or (2) with other critical illnesses will be excluded.

\section{Interventions}

The control group subjects will have received routine treatment, which refers to interactions between nurses and patients without intentionally connecting patients with their peers. The intervention groups will have been intentionally connected with their peers or/and enrolled in peer support training.

\section{Outcomes}

Primary outcomes:

- Scores on an anxiety scale (Self-Rating Anxiety Scale $(\mathrm{SAS})^{27}$ or Hamilton Anxiety Scale).$^{28}$

Secondary outcomes:

- Heart rate.

- Blood pressure.

\section{Search strategy and identification of studies}

\section{Electronic searches}

We will search for articles in these databases: PubMed, EMBASE, Cochrane Library, CINAHL, Chinese National Knowledge Infrastructure, Wanfang Data, Chinese BioMedical Database and Chinese Science and Technology Periodicals Database. The search period will be from the date of database inception to January 2019. The key terms for the PubMed search are shown in figure 1.

\section{Searching other resources}

We will search Clinical trials.gov and the WHO International Clinical Trials Registry Platform Search Portal to

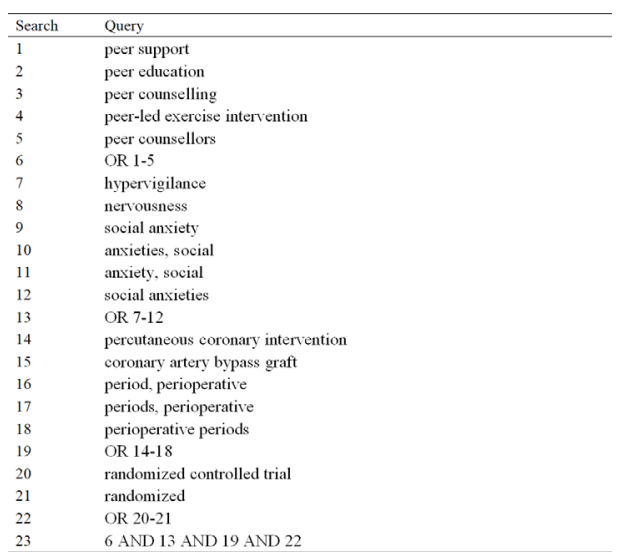

Figure 1 Key terms for the PubMed search. 


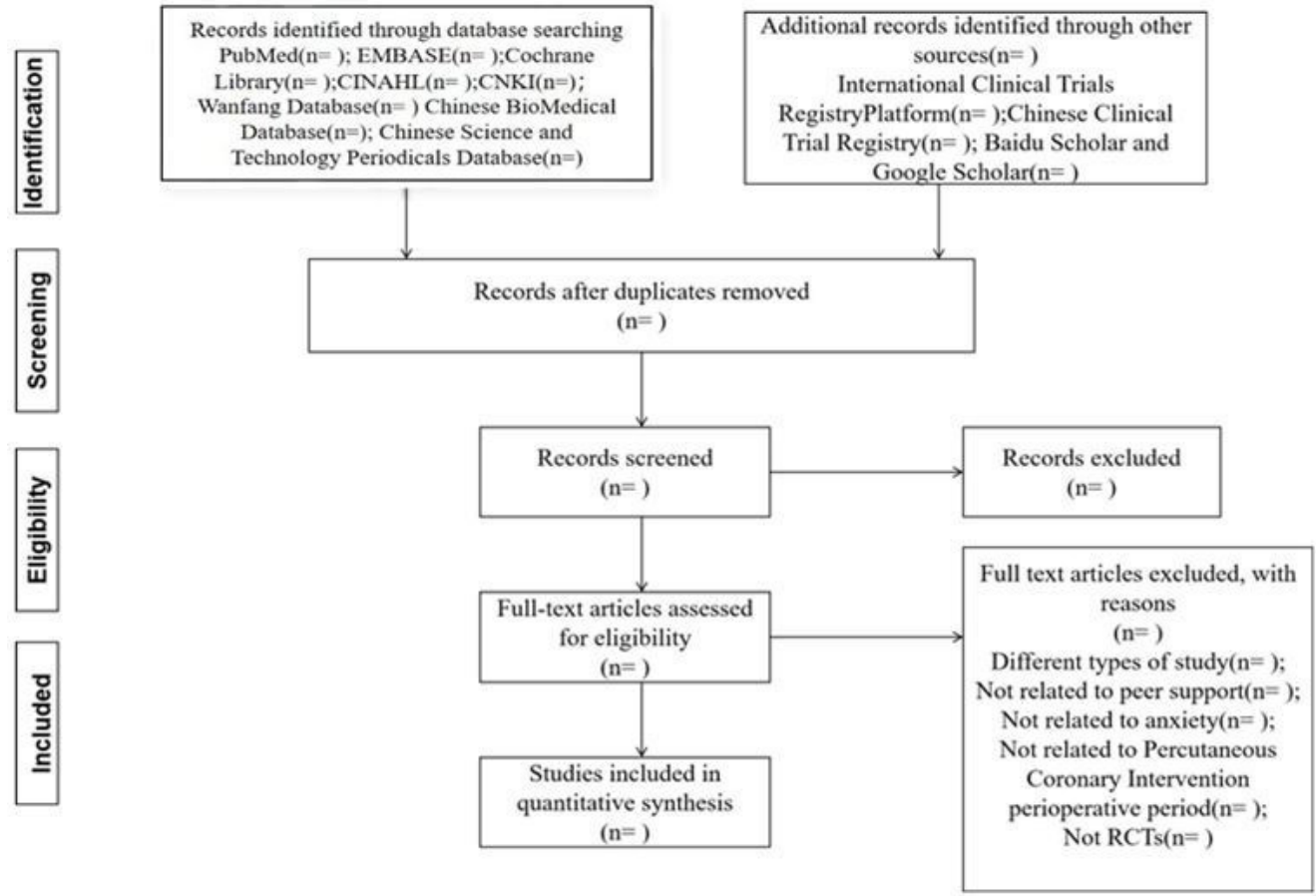

Figure 2 Flow diagram of the study selection process. RCTs, randomised controlled trials.

find ongoing studies. The grey literature will be searched through Baidu Scholar and Google Scholar. We will use Chinese translations of these key words for the Chinese databases. We will contact the authors to obtain further information, if necessary. Study selection will be based on the PRISMA flow chart (http://www.prisma-statement. org) (figure 2).

\section{Data management}

NoteExpress software will be used to manage all the initially identified literature and remove duplications. Two reviewers (SQ and TS) will independently review the full texts of the selected articles and use a researcherdeveloped form to extract information about the research population, interventions and outcomes. The form will include the following items: general information, test characteristics, participants, interventions and outcomes. If necessary, differences of opinion will be discussed with the third author (YG).

\section{Addressing missing data or unclear measurement scales}

If necessary, to process missing data or unclear measurement scales, we will contact the author of the article via email or telephone. If this is not possible, we will analyse the effectiveness using the available information.

\section{Risk of bias in included studies}

Two independent reviewers (SQ and TS) will use the Cochrane Handbook for Systematic Reviews of Interventions to evaluate the risk of bias of the included articles. The following six domains will be assessed: random sequence generation, allocation concealment, blinding of participants and personnel, blinding of outcome assessment, incomplete outcome data, selective reporting and other bias. We will classify the included studies into three categories (low risk of bias, high risk of bias and unclear risk of bias). If an agreement cannot be reached, the third investigator $(\mathrm{YG})$ will provide a suggestion.

\section{Data synthesis}

RevMan V.5.3.5 software will be used for the analyses.

\section{Treatment effect measure}

Dichotomous outcomes (mortality, hospital readmission and so on) will be assessed using risk ratios with $95 \%$ CIs, and continuous outcomes (Self-Rating Depression Scale and so on) will be described with the weighted mean difference or standardised mean difference with $95 \%$ CIs. For the assessment of heterogeneity, the $\mathrm{Q}$ and $\mathrm{I}^{2}$ statistics will be used. If the included studies have $\mathrm{p}<0.05$, the difference will be considered significant. In addition, $\mathrm{I}^{2}<25 \%$ will indicate low significant heterogeneity, $25 \%<\mathrm{I}^{2}<50 \%$ will indicate moderate heterogeneity, and $\mathrm{I}^{2}>50 \%$ will indicate strong heterogeneity. The fixedeffects model will be used to assess studies that are not heterogeneous, and the random-effects model will be used to assess heterogeneous studies.

\section{Additional analysis}

We will conduct an analysis based on the different research characteristics. If data extraction is insufficient, we will perform a qualitative synthesis.

\section{Assessment of reporting bias}

A funnel plot will be developed to assess reporting bias. We will use Begg's and Egger's tests to evaluate the symmetry of the funnel graph, and numerical values of $\mathrm{p}<0.1$ will indicate statistical significance. 


\section{Patient and public involvement statement}

We have invited a number of physicians who specialise in cardiovascular medicine to help us refining our research issues and primary and secondary outcomes. However, they were not involved in the design of any other aspects of the study or in the drafting of the protocol. Due to the nature of the study design that we propose, neither patients nor the public will be involved in the design or implementation of the study.

\section{ETHICS AND DISSEMINATION}

No ethics approval will be required because this is a systematic review of published studies. The current study includes results from articles that have been published previously. The results of this study will be published in an open access journal without any profit for the authors.

\section{DISCUSSION}

The anxiety level of patients before coronary angiography or PCI is higher than that of healthy people not undergoing these procedures (14\%). ${ }^{1}$ The SAS and Hamilton's Anxiety Scale (HAMA) have been used to evaluate perioperative anxiety in many studies. ${ }^{911} 13-16$ (1) The SAS was developed by William W K Zung and is one of the most commonly used psychological measurement tools. ${ }^{27} \mathrm{~A}$ standard score $>50$ indicates the presence of anxiety symptoms. (2) HAMA is the other anxiety rating scale, which was generated by Hamilton in 1959. HAMA is a commonly used scale in psychiatric clinics. ${ }^{28}$ A score $\geq 29$ indicates severe anxiety; a score $\geq 21$ indicates moderate anxiety; a score $\geq 14$ indicates mild anxiety; a score $\geq 7$ indicates possible anxiety; and a score $<7$ indicates no anxiety. Seventy per cent of patients with PCI are diagnosed with anxiety, ${ }^{9}$ which directly leads to the occurrence of cardiovascular events. Drug and non-drug methods can be used to alleviate anxiety. Compared with drugs, nondrug methods have fewer side effects and lesser risk and are more easily implemented; non-drug methods are relatively safer and non-invasive. Helping patients understand coronary heart disease, and the details of surgery can alleviate their anxiety and enhance their compliance. In recent years, some studies have introduced the peer support model into the field of psychological nursing for patients with coronary heart disease during the PCI perioperative period. The incidence of coronary heart disease is related to poor living habits and poor treatment compliance. Esmaeili $e t a l^{29}$ believe that one appropriate non-drug method for reducing anxiety levels is the provision of adequate information on disease control and care and follow-up plans by knowledgeable people with experience with the same disease. Some foreign scholars have applied the non-drug psychological intervention nursing method to relieve anxiety after cardiac surgery. Lai et $a l^{30}$ suggested that patients and their families are unfamiliar with cardiac surgery and its complications, which leads to postoperative anxiety. Providing postoperative nursing information to patients and their families could effectively reduce anxiety. Beibei $e t a l^{\beta 1}$ proposed that peer education can significantly improve the self-management efficiency of patients after PCI, reduce medical costs, and reduce the associated social burden. It is an effective form of health education. Wu $e t a \hat{l}^{32}$ conducted an RCT of 68 patients and found that peer support can significantly improve patient compliance and reduce negative emotions. Varaei et $a l^{19}$ noted that peer support improved the self-efficacy of the subjects and reduced the rehospitalisation rate within 8 months after discharge. The sharing of experiences of positive coping with disease among patients helps increasing patients' confidence in their self-management skills and promotes their compliance with disease treatment. Because peer communication is convenient and accessible, there is hope that it can effectively improve patients' confidence in their health management abilities; however, there is still a need for a sufficient sample size to illustrate whether peer support can reduce perioperative anxiety in patients with PCI. This study will provide strong evidence by means of a meta-analysis and systematic review regarding the ability of peer support to reduce perioperative anxiety in patients scheduled to undergo coronary angiography or PCI.

Acknowledgements We would like to offer our special thanks to American Journal Experts for dedicating their time to editing our protocol.

Contributors SQ formulated the literature retrieval strategy and data analysis strategies and wrote the article; TS participated in the formulation of the literature retrieval strategy; YG improved and revised the article.

Funding This work was supported by the Humanities and Social Sciences Project of Hebei Provincial Education Department, grant number GH151054.

Competing interests None declared.

Patient and public involvement Patients and/or the public were not involved in the design, or conduct, or reporting, or dissemination plans of this research.

Patient consent for publication Not required.

Provenance and peer review Not commissioned; externally peer reviewed.

Open access This is an open access article distributed in accordance with the Creative Commons Attribution Non Commercial (CC BY-NC 4.0) license, which permits others to distribute, remix, adapt, build upon this work non-commercially, and license their derivative works on different terms, provided the original work is properly cited, appropriate credit is given, any changes made indicated, and the use is non-commercial. See: http://creativecommons.org/licenses/by-nc/4.0/.

ORCID iD

Shuo Qin http://orcid.org/0000-0002-2505-4211

\section{REFERENCES}

1 Piccolo R, Giustino G, Mehran R, et al. Stable coronary artery disease: revascularisation and invasive strategies. Lancet 2015;386:702-13.

2 Gorog D. Coronary heart disease, 2018.

3 Wei Z. The relationship between glycosylated hemoglobin, lipoprotein $A$ and the severity of coronary artery disease in patients with coronary heart disease. Zhengzhou University, 2014.

4 Acharjee S, Teo KK, Jacobs AK, et al. Optimal medical therapy with or without percutaneous coronary intervention in women with stable coronary disease: a pre-specified subset analysis of the clinical outcomes utilizing revascularization and aggressive drug evaluation (courage) trial.. Am Heart J 2016;173:108-17.

5 Peking Union Medical University. Statistical Yearbook of health and family planning in China. Publishing House, Peking Union Medical University, 2013. 
6 Maliyuan WY, Wen W, Weiwei C. Essential interpretation of China cardiovascular disease report 2017. Chin J Cardiol 2018;23:3-6.

7 Danni X, Chenxi X, Li Biao XX. Distribution and inspiration of medical institutions and doctors in China's online medical services. China's health management 2018.

8 Dorje T, Zhao G, Scheer A, et al. SMART phone and social mediabased cardiac rehabilitation and secondary prevention (SMART-CR/ $\mathrm{SP})$ for patients with coronary heart disease in China: a randomised controlled trial protocol. BMJ Open 2018;8:e21908.

9 Xian W, Zhu Q, Zhifu Z. Expert consensus on diagnosis and treatment of depression and/or anxiety before and after percutaneous coronary intervention (PCI). Journal of Traditional Chinese Medicine 2015;4:357-60.

10 Xueqin L, Wenjuan H. Self-management behavior of patients after percutaneous coronary intervention and its correlation with adverse cardiovascular events. Practical preventive medicine 2018;25:1113-6.

11 Uzun S, Vural H, Uzun M, et al. State and trait anxiety levels before coronary angiography. J Clin Nurs 2008;17:602-7.

12 Lina $\mathrm{H}$. Application value of continuing nursing intervention in post$\mathrm{PCl}$ nursing of patients with acute myocardial infarction. Chinese Medical Guidelines 2017;15:240-1.

13 Habibzadeh H, Milan ZD, Radfar M, et al. Effects of Peer-Facilitated, video-based and combined Peer-and-Video education on anxiety among patients undergoing coronary angiography: randomised controlled trial. Sultan Qaboos Univ Med J 2018;18:61-6.

14 Tahmasbi H, Mahmoodi G, Mokhberi V, et al. The impact of Aromatherapy on the anxiety of patients experiencing coronary angiography. Zahedan Journal of Research in Medical Sciences 2012;14.

15 Abdi $\mathrm{H}$, Hejazi S, Tahmasebi $\mathrm{H}$, et al. Effect of AROMATHERAPY with ORANG E essential oils on anxiety in patients experiencing coronary angiography: a randomized control trial. Journal Of Urmia Nursing And Midwifery Faculty 2018;15.

16 Homeyra T, Soghra H, Homeyra A, et al. Trait anxiety and state anxiety before E E coronary angiography. Journal Of Health Breeze 2012;1:41-6.

17 Tiehan W, Wang Yichao LA. The effect of cognitive emotion on blood pressure and heart rate in patients with hypertension and drug intervention research. China Health Industry 2012;14:106-7.

18 Højskov IE, Moons P, Hansen NV, et al. SheppHeartCABG trialcomprehensive early rehabilitation after coronary artery bypass grafting: a protocol for a randomised clinical trial. BMJ Open 2017;7:e13038.

19 Varaei S, Shamsizadeh M, Cheraghi MA, et al. Effects of a peer education on cardiac self-efficacy and readmissions in patients undergoing coronary artery bypass graft surgery: a randomizedcontrolled trial. Nurs Crit Care 2017:22:19-28.

20 Nankabirwa V, Tylleskar T, Nankunda J, et al. Malaria parasitaemia among infants and its association with breastfeeding peer counselling and vitamin A supplementation: a secondary analysis of a cluster randomized trial. PLoS One 2011;6:e21862.

21 Kumakech E, Cantor-Graae E, Maling S, et al. Peer-group support intervention improves the psychosocial well-being of AIDS orphans: cluster randomized trial. Soc Sci Med 2009;68:1038-43.

22 Chunyan Y. Practical study on the effect of relaxation massage on stress response of patients before percutaneous coronary intervention. Shanxi Medical University, 2012.

23 Yuhong J, Yehong Q, Yan Z. Analysis of the effect of cognitive behavioral intervention on anxiety and depression in patients after coronary artery stent implantation. Journal of Bengbu Medical College 2017.

24 Juan Z. The effect of preoperative video education on anxiety and surgical tolerance of patients with PCl. Qilu Journal of Nursing 2014;20:90-1.

25 Jianhui W, Wenhong C, Jing $\mathrm{H}$. The effect of music intervention on perioperative anxiety in patients with coronary heart disease undergoing PCI. Nursing research 2012;26:520-1.

26 Meijuan Q. The influence of evidence-based nursing concept on the prognosis and quality of life of patients with acute coronary syndrome. Chinese and foreign women's health research 2017:19:157-9.

27 Li H, Jin D, Qiao F, et al. Relationship between the self-rating anxiety scale score and the success rate of 64-slice computed tomography coronary angiography. Int J Psychiatry Med 2016;51:47-55.

28 Snaith RP, Baugh SJ, Clayden AD, et al. The clinical anxiety scale: an instrument derived from the Hamilton anxiety scale. $\mathrm{Br} J$ Psychiatry 1982;141:518-23.

29 Esmaeili R, Jannati Y, Ghafari R, et al. A clinical trial comparing the effect of peer education and orientation program on the anxiety levels of pre-CABG surgery patients. J Med Life 2015;8:66.

30 Lai VKW, Lee A, Leung P, et al. Patient and family satisfaction levels in the intensive care unit after elective cardiac surgery: study protocol for a randomised controlled trial of a preoperative patient education intervention. BMJ Open 2016;6:e11341.

31 Beibei W, Wenjie Z, Yan Z, et al. Application of peer education in self-management of patients with coronary heart disease after $\mathrm{PCl}$ Nursing practice and research 2017.

32 Wu J, Wang C, Du L. The effect of peer education on anxiety and depression in patients with coronary heart disease after PCl. Chinese community physician 2017;17:147-8. 